\title{
Approximation of First Grade MHD Squeezing Fluid Flow with Slip Boundary Condition Using DTM and OHAM
}

\author{
Inayat Ullah, Hamid Khan, and M. T. Rahim \\ Department of Mathematics, National University of Computer and Emerging Sciences, \\ FAST Peshawar Campus, Peshawar 25000, Pakistan
}

Correspondence should be addressed to Inayat Ullah; inayat_28@hotmail.com

Received 27 December 2012; Accepted 24 April 2013

Academic Editor: Safa Bozkurt Coskun

Copyright (C) 2013 Inayat Ullah et al. This is an open access article distributed under the Creative Commons Attribution License, which permits unrestricted use, distribution, and reproduction in any medium, provided the original work is properly cited.

A steady two-dimensional axisymmetric flow of an incompressible viscous fluid under the influence of a uniform transverse magnetic field with slip boundary condition is studied. An ordinary nonlinear differential equation is formed by transforming the Navier-Stokes equations using the transformation $\psi(r, z)=r^{2} G(z)$. Differential transform and optimal homotopy analysis methods have been used to obtain the solutions by varying pertinent flow parameters. By using residuals in each case, the validity of solutions is established. Excellent results are obtained by using the proposed schemes. The influence of different parameters on the flow is shown through graphs.

\section{Introduction}

Squeezing flows induce when normal stresses or vertical velocities are applied externally by means of a mobile boundary [1]. Stefan [2] carried out the fundamental research in this field. Engineers studied the analysis of Newtonian fluid squeezed between two infinite planar plates $[3,4]$. There are many applications of squeezing flow in food industry, especially in chemical engineering [4]. Polymer processing, compression, and injection modeling are the practical examples of squeezing flow. Thin Newtonian liquid films squeezing between two plates were studied by Grimm [5]. Squeezing flow under the influence of magnetic field is widely applied to bearing with liquid-metal lubrication [6-9]. Islam et al. [10] studied squeezing fluid flow between the two infinite parallel plates in a porous medium channel.

In case of many polymeric liquids when the weight of molecule is high, then they show slip at the boundary. The no-slip boundary condition is not applicable in this case. In many cases such as thin film problems, rarefied fluid problems, fluids containing concentrated suspensions, and flow on multiple interfaces, the no-slip boundary condition fails to work. Navier [11], for the first time, proposed the general boundary condition which demonstrates the fluid slip at the surface. The difference of fluid velocity and velocity of the boundary is proportional to the shear stress at that boundary. The proportionality constant is named the slip parameter having length as its dimension.

The slip condition is of great importance especially when fluids with elastic character are under consideration [12]. Newtonian fluid was considered by Ebaid [13] to study the effects of magnetic field and wall slip conditions on the peristaltic transport in an asymmetric channel. It has great importance in medical sciences, particularly in polishing artificial heart valves and internal cavities in many manufactured parts achieved by embedding such fluids with abrasives [14]. The influence of slip on the peristaltic motion of third-order fluid in asymmetric channel is studied by Hayat et al. [15]. The effects of slip condition on the rotating flow of a third grade fluid in a nonporous medium are investigated by Hayat and Abelman [16]. The extension of the work in [16] to a porous medium and obtaining the numerical solutions for the steady magnetohydrodynamics flow of a third grade fluid in a rotating frame is presented by Abelman et al. [17].

Various perturbation techniques can be applied to solve nonlinear differential equations [18]. The limitations of these methods are based on the assumption of small parameter and there is no proper way to select this parameter. 


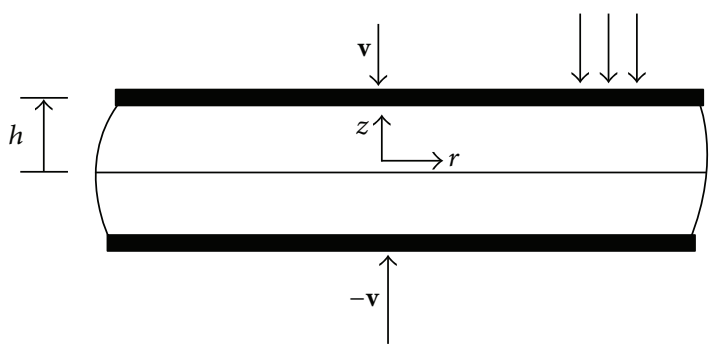

FIGURE 1: A steady squeezing axisymmetric fluid flow between two parallel plates.

The idea of homotopy was combined with perturbation in the last decade. Liao and He did the fundamental work on it. Homotopy analysis method (HAM) was proposed by Liao [19-21]. Homotopy perturbation method was introduced by He [22-24]. Marinca et al. [25, 26] introduced OHAM for approximate solution of nonlinear problems of thin film flow of a fourth-grade fluid down a vertical cylinder and for the study of the behavior of nonlinear mechanical vibration of electrical machines. It is observed that HPM and HAM are the special cases of OHAM [27, 28].

Differential transform method (DTM) was initially introduced by Zhou in 1986 [29]. Çatal [30-33] studied solution of free vibration equations of beam on elastic soil, buckling analysis of partially embedded pile in elastic soil, analysis of free vibration of beam on elastic soil, and response of forced Euler-Bernoulli beams by using DTM. Ayaz [34] applied this method to differential algebraic equations (DAEs) of index1. Liu and Song [35] investigated that DTM is effective in case of index-2 DAEs but not in index-3. Ayaz [36] studied the applications of two-dimensional DTM in case of partial differential equations.

The objective of this research paper is to use OHAM and DTM for studying MHD squeezing flow with slip boundary condition between two infinite plates approaching each other slowly. In Section 2, the basic equations are derived and the model is reduced to a nonlinear boundary value problem. In Sections 3 and 4, the basic ideas of DTM and its use for our problem are presented while the idea of OHAM and its application to our problem are in Sections 5 and 6, respectively.

\section{Problem Formulation}

Let us consider, in the presence of a magnetic field, a squeezing flow of an incompressible Newtonian fluid with constant density $\rho$ and viscosity $\mu$, squeezed between two large planar parallel plates separated by a small distance $2 h$ approaching each other with a low constant velocity $\mathbf{v}$ (Figure 1 ). Assume that the flow is quasisteady $[1,3]$, and the NavierStokes equations governing such flow when inertial terms are retained are

$$
\begin{gathered}
\nabla \cdot \mathbf{v}=0 \\
\rho\left[\frac{\partial \mathbf{v}}{\partial}+(\nabla \cdot \mathbf{v}) \mathbf{v}\right]=\nabla \cdot \mathbf{T}+(\mathbf{v} \times \mathbf{B}) \times \mathbf{B}
\end{gathered}
$$

where $\nabla$ denotes the material time derivative, $\mathbf{T}$ is the Cauchy stress tensor given by $\mathbf{T}=-p I+\mu A$ with $A=\nabla \mathbf{v}+(\mathbf{v})^{t}$. $\mathbf{B}$ is the total magnetic field given by $\mathbf{B}=\mathbf{B}_{0}+b . \mathbf{B}_{0}$ and $b$ represent the imposed and induced magnetic fields, respectively. The modified Ohm's law and Maxwell's equations (see [37] and the references therein), in the absence of displacement currents, are

$$
\begin{array}{lr}
\mathbf{J}=\sigma[E+\mathbf{v} \times \mathbf{B}], & \nabla \cdot \mathbf{B}=0, \\
\nabla \times \mathbf{B}=\mu_{m} \mathbf{J}, & \operatorname{curl} E=\frac{\partial \mathbf{B}}{\partial t} .
\end{array}
$$

Here $\mathbf{J}$ is the electric current density, $\sigma$ represents the electrical conductivity, $E$ the electric field, and $\mu_{m}$ the magnetic permeability. If $\rho, \mu_{m}$, and $\sigma$ are constant, $b$ is negligible as compared to $\mathbf{B}_{0}, \mathbf{B}$ is perpendicular to $\mathbf{v}$ so that the Reynold number is small with no electric field in the fluid flow region and then the magneto hydrodynamic force involved can be written as

$$
\mathbf{J} \times \mathbf{B}=-\sigma \mathbf{B}_{0}^{2} \mathbf{v}
$$

Assuming that the plates are nonconducting and the magnetic field is applied along the $z$-axis. The gap distance $2 h$ between the plates changes slowly with time $t$ for small values of the velocity $\mathbf{v}$ so that it can be taken as constant. An axisymmetric flow in cylindrical coordinates $(r, \theta, z)$ with $z$ axis perpendicular to plates and $z= \pm h$ at the plates. For axial symmetry, $v$ is represented by $v=\left(v_{r}, 0, v_{z}\right)$. In view of negligible body forces with no tangential velocity, NavierStokes equations $[1,6,10]$ in cylindrical coordinates are

$$
\begin{gathered}
\frac{\partial p}{\partial r}-\rho \Omega \mathbf{v}_{z}=-\mu \frac{\partial \Omega}{\partial z}-\sigma \mathbf{B}_{0}^{2} \mathbf{v}_{r}, \\
\frac{\partial p}{\partial z}+\rho \Omega \mathbf{v}_{r}=\frac{\mu}{r} \frac{\partial}{\partial r}(r \Omega),
\end{gathered}
$$

where

$$
\Omega(r, z)=\frac{\partial \mathbf{v}_{z}}{\partial r}-\frac{\partial \mathbf{v}_{r}}{\partial z}
$$

Introducing the stream function $\psi(r, z)$, we have,

$$
\mathbf{v}_{r}=\frac{1}{r} \frac{\partial \psi}{\partial z}, \quad \mathbf{v}_{z}=-\frac{1}{r} \frac{\partial \psi}{\partial r} .
$$

Eliminating $p$ from (4), we have

$$
\rho\left[\frac{\partial\left(\psi, E^{2} \psi / r^{2}\right)}{\partial(r, z)}\right]=-\frac{\mu}{r} E^{4} \psi+\frac{\sigma \mathbf{B}_{0}^{2}}{r} \frac{\partial^{2} \psi}{\partial z^{2}},
$$

where

$$
E^{2}=\frac{\partial^{2}}{\partial r^{2}}-\frac{1}{r} \frac{\partial}{\partial r}+\frac{\partial^{2}}{\partial z^{2}}
$$

Using the transformation $\psi(r, z)=r^{2} g(z)$, (7) can be written as

$$
g^{(i v)}(z)-\frac{\sigma \mathbf{B}_{0}^{2}}{\mu} g^{\prime \prime}(z)+2 \frac{\rho}{\mu} g(Z) g^{\prime \prime \prime}(z)=0,
$$


subject to the slip boundary conditions

$$
\begin{gathered}
g(0)=0, \quad g^{\prime \prime}(0)=0, \\
g(h)=\frac{v}{2}, \quad g^{\prime}(h)=\beta g^{\prime \prime}(h) .
\end{gathered}
$$

The nondimensional parameters are $G^{*}=g / v / 2, z^{*}=z / h$, $R=\rho h / \mu / \nu$, and $m=\mathbf{B}_{0} h \sqrt{\sigma / \mu}$. Omitting the ${ }^{*},(9)$ and (10) become

$$
\begin{gathered}
G^{(i v)}(z)-m^{2} G^{\prime \prime}(z)+R G(z) G^{\prime \prime \prime}(z)=0, \\
G(0)=0, \quad G^{\prime \prime}(0)=0 \\
G(1)=1, \quad G^{\prime}(1)=\gamma G^{\prime \prime}(1),
\end{gathered}
$$

with $\gamma=\beta / h$ and $R, m$ are the Reynolds and Hartmann numbers, respectively.

\section{Differential Transform Method}

One-dimensional differential transform of a function $g(z)$ is defined as follows [29-35]:

$$
G(z)=\frac{1}{k !}\left[\frac{d^{k} g(z)}{d z^{k}}\right]_{z=0} .
$$

$G(z)$ is also called the T-function. The inverse transform of $G(z)$ is is defined as

$$
g(z)=\sum_{k=0}^{\infty} z^{k} G(z) .
$$

Combining (12) and (13), we can write

$$
g(z)=\sum_{k=0}^{\infty} \frac{z^{k}}{k !}\left[\frac{d^{k} g(z)}{d z^{k}}\right] .
$$

Usually we express $g(z)$ in a finite series, that is,

$$
g(z)=\sum_{k=0}^{N} \frac{z^{k}}{k !}\left[\frac{d^{k} g(z)}{d z^{k}}\right],
$$

which implies that

$$
g(z)=\sum_{k=N+1}^{\infty} \frac{z^{k}}{k !}\left[\frac{d^{k} g(z)}{d z^{k}}\right]
$$

is negligibly small.

Some fundamental theorems on one-dimensional differential transform are as follows.

Theorem 1. If $g(z)=f(z) \pm y(z)$, then $G(k)=F(k) \pm Y(k)$.

Theorem 2. If $g(z)=y^{(n)}(z)$, then $G(z)=((k+n) ! / k !) Y(k+$ $n)$.

Theorem 3. If $g(z)=y(z) \cdot f(z)$, then $G(z)=\sum_{r=0}^{k} Y(r) F(k-$ $r)$.

Theorem 4. If $g(z)=z^{n}$, then

$$
G(z)=\delta(k-n)= \begin{cases}1, & \text { if } k=n, \\ 0, & \text { if } k \neq n .\end{cases}
$$

\section{Analysis of Differential Transform Method}

For fourth-order boundary value problem [10],

$$
g^{(i v)}=y(z, g)
$$

with boundary conditions

$g(0)=\eta_{0}, \quad g(h)=\eta_{1}, \quad g^{\prime}(h)=\eta_{2}, \quad g^{\prime \prime}(0)=\eta_{3}$.

The differential transform is given by

$$
G(k+4)=\frac{Y(k)}{\prod_{j=1}^{4}(k+j)},
$$

with transformed boundary conditions

$$
\begin{gathered}
G(0)=\eta_{0}, \quad \sum_{k=0}^{N} h^{k} G(k)=\eta_{1}, \\
\sum_{k=0}^{N} k h^{k-1} G(k)=\eta_{2}, \quad G(2)=\frac{\eta_{3}}{2} .
\end{gathered}
$$

Using (20) and (21), $G(j)$ for $j=4,5, \ldots$ is evaluated to obtain the solution up to $O\left(z^{N+1}\right)$ as follows:

$$
g(z)=\sum_{k=0}^{N} z^{k} G(k)+O\left(z^{N+1}\right) .
$$

\section{Application of DTM to Our Problem}

Here, we consider an incompressible Newtonian fluid, squeezed between two large planar, parallel plates which are separated by a small distance $2 h$ and moving towards each other with velocity $\mathbf{v}$. For small values of $\mathbf{v}$, the gap distance $2 h$ between the plates changes slowly with time $t$, so that it can be taken as constant, and the flow is steady as

$$
G^{(i v)}(z)-m^{2} G^{\prime \prime}(z)+R G(z) G^{\prime \prime \prime}(z)=0,
$$

with slip boundary conditions

$$
\begin{gathered}
G(0)=0, \quad G^{\prime \prime}(0)=0, \\
G(1)=1, \quad G^{\prime}(1)=\gamma G^{\prime \prime}(1) .
\end{gathered}
$$

The differential transform of (23) is given by

$$
\begin{aligned}
& \widetilde{G}(k+4) \\
& =\frac{k !}{(k+4) !} \\
& \quad \times\left\{\begin{array}{l}
m^{2}(k+1)(k+2) \widetilde{G}(k+2) \\
-R \sum_{r=0}^{k}(r+1)(r+2)(r+3) \widetilde{G}(r+3) \widetilde{G}(k-r),
\end{array}\right.
\end{aligned}
$$


with transformed boundary conditions

$$
\widetilde{G}(0)=0, \quad \widetilde{G}(1)=a, \quad \widetilde{G}(2)=0, \quad \widetilde{G}(3)=b,
$$

where $a$ and $b$ are unknowns to be determined later [38]. Using (25) and (26), the values of $\widetilde{G}(i), i=1,2,3, \ldots, 15$ are

$$
\begin{aligned}
& \widetilde{G}(2 k)=0, \quad \text { for } k=0(1) 7, \\
& \widetilde{G}(5)=\frac{1}{120}\left(6 b m^{2}-6 a b R\right), \\
& \widetilde{G}(7)=\frac{1}{840 b}\left(m^{4}-4 a m^{2} R+3 R\left(-2 b+a^{2} R\right)\right), \\
& \widetilde{G}(9)=\frac{b}{60480} \\
& \times\left(m^{6}-9 a m^{4} R+3 a R^{2}\left(32 b-5 a^{2} R\right)\right. \\
& \left.+m^{2} R\left(-72 b+23 a^{2} R\right)\right), \\
& \widetilde{G}(11)=\frac{b}{6652800} \\
& \times\left\{\begin{array}{l}
m^{8}-16 a m^{6} R-44 a m^{2} R^{2}\left(-39 b+4 a^{2} R\right) \\
+m^{4}\left(-414 b R+86 a^{2} R^{2}\right) \\
+3 R^{2}\left(432 b^{2}-482 a^{2} b R+35 a^{4} R^{2}\right),
\end{array}\right. \\
& \widetilde{G}(13)=\frac{b}{1037836800} \\
& \times\left\{\begin{array}{l}
m^{10}-25 a m^{8} R \\
+2 a m^{4} R^{2}\left(7446 b-475 a^{2} R\right) \\
+2 m^{6} R\left(-948 b+115 a^{2} R\right) \\
-9 a R^{3}\left(7384 b^{2}-2620 a^{2} b R+105 a^{4} R^{2}\right) \\
+3 m^{2} R^{2}\left(14616 b^{2}-11808 a^{2} b R\right. \\
\left.+563 a^{4} R^{2}\right),
\end{array}\right.
\end{aligned}
$$$$
\widetilde{G}(15)=\frac{b}{217945728000}
$$$$
\times\left\{\begin{array}{c}
m^{12}-36 a m^{10} R \\
+24 a m^{6} R^{2}\left(4106 b-145 a^{2} R\right) \\
+m^{8} R\left(-7974 b+505 a^{2} R\right) \\
-12 a m^{2} R^{3}\left(239508 b^{2}-62828 a^{2} b R\right. \\
\left.+1627 a^{4} R^{2}\right) \\
+m^{4} R^{2}\left(703296 b^{2}-427716 a^{2} b R\right. \\
\left.+12139 a^{4} R^{2}\right) \\
+9 R^{3}\left(-151344 b^{3}+282256 a^{2} b^{2} R\right. \\
\left.-47590 a^{4} b R^{2}+1155 a^{6} R^{3}\right)
\end{array}\right.
$$

As $R, m$ have been nondimensionalized, so let us fix them one to find the values of $a$ and $b$ from the following system:

$$
\begin{gathered}
\sum_{k=0}^{15}\left(k(h)^{k-1} \widetilde{G}[k]-k(k-1)(h)^{k-2} \widetilde{G}[k]\right)=0, \\
\sum_{k=0}^{15}(h)^{k} \widetilde{G}[k]=1,
\end{gathered}
$$

which leads us to the following values:

$$
a=0.754966, \quad b=0.242565 \text {. }
$$

Using (22), the approximate solution of the BVP is as follows:

$$
\begin{aligned}
& G(z) \\
& =\left\{\begin{array}{l}
0.754966 z+0.242565 z^{3}+0.00297182 z^{5} \\
-0.00050977 z^{7} \\
+3.913152541684915 \times 10^{-6} z^{9} \\
+3.1529964918115006 \times 10^{-6} z^{11} \\
-1.2149332568415323 \times 10^{-7} z^{13} \\
-2.0892317176411676 \times 10^{-8} z^{15}+O\left(z^{16}\right) .
\end{array}\right.
\end{aligned}
$$

This is a series solution and we note that the terms approach to zero if we go forward which is the beauty of DTM and other series solutions.

\section{Basic Idea of OHAM}

Let us consider a boundary value problem [25-27]

$$
\zeta[g(z)]+f(z)+\eta[g(z)]=0
$$

along with boundary conditions

$$
B\left(g, \frac{d g}{d z}\right)=0
$$

where $z$ denotes the independent variable, $g(z), f(z)$ are unknown and known functions and $\zeta, \eta, B$ are linear, nonlinear, and boundary operators, respectively.

According to OHAM a homotopy, $h(G(z, p), p): \mathbf{R} \times$ $[0,1] \rightarrow \mathbf{R}$ satisfying

$$
\begin{aligned}
& (1-p)[\zeta(G(z, p))+f(z)] \\
& =h(p)[\zeta(G(z, p))+f(z)+\eta(G(z, p))], \\
& B\left(G(z, p), \frac{\partial G(z, p)}{\partial z}\right)=0
\end{aligned}
$$

for $z \in R$ and $p \in[0,1]$, is constructed with nonzero auxiliary function $h(p)$ for $p \neq 0$ with $h(0)=0$ and for an unknown function $G(z, p)$. For $p=0,1$ the equalities $G(z, 0)=g_{0}(z)$ and $G(z, 1)=g(z)$ hold. So it is concluded that the solution 
$G(z, p)$ approaches from $g_{0}(z)$ to $g(z)$ as $p$ varies from 0 to 1 . For $p=0$,

$$
\zeta\left(g_{0}(z)\right)+f(z)=0, \quad B\left(g_{0}, \frac{d g_{0}}{d z}\right)=0 .
$$

We choose the auxiliary function $h(p)$ such that

$$
h(p)=\sum_{k=0}^{n} p^{k} C_{k}
$$

where $C_{k}$ are the convergence controlling constants to be determined. Expanding $G(z, p)$ in Taylor's series about $p$ to get the approximation

$$
G\left(z, p, C_{k}\right)=g_{0}(z)+\sum_{j=1}^{n} g_{j}\left(z, C_{1}, C_{2}, \ldots, C_{j}\right) p^{j},
$$

substituting (36) into (33), and equating the coefficients of like powers of $p$, we get the following.

Zeroth-order problem is

$$
\zeta\left(g_{0}(z)\right)+f(z)=0, \quad B\left(g_{0}, \frac{d g_{0}}{d z}\right)=0 .
$$

First-order problem is

$$
\zeta\left(g_{1}(z)\right)+f(z)=C_{1} \eta_{0}\left(g_{0}(z)\right), \quad B\left(g_{1}, \frac{d g_{1}}{d z}\right)=0 .
$$

Second-order problem is

$$
\begin{aligned}
& \zeta\left(g_{2}(z)\right)-\zeta\left(g_{1}(z)\right) \\
& =\left\{\begin{array}{l}
C_{2} \eta_{0}\left(g_{0}(z)\right)+C_{1}\left[\zeta\left(g_{1}(z)\right)+\eta_{1}\left(g_{0}(z), g_{1}(z)\right)\right] \\
B\left(g_{2}, \frac{d g_{2}}{d z}\right)=0 .
\end{array}\right.
\end{aligned}
$$

Generally,

$$
\begin{aligned}
& \zeta\left(g_{n}(z)\right)-\zeta\left(g_{n-1}(z)\right)= \\
& \left\{\begin{array}{l}
C_{n} \eta_{0}\left(g_{0}(z)\right) \\
+\left(\sum _ { j = 1 } ^ { n - 1 } C _ { j } \left[\zeta\left(g_{n-j}(z)\right)\right.\right. \\
\left.\left.\quad+\eta_{n-j}\left(g_{0}(z), g_{1}(z), \ldots, g_{n-1}(z)\right)\right]\right),
\end{array}\right.
\end{aligned}
$$

with boundary conditions

$$
B\left(g_{k}, \frac{d g_{k}}{d z}\right)=0, \quad k=2,3,4, \ldots
$$

where $\eta_{m}\left(g_{0}(z), g_{1}(z), \ldots, g_{m-1}(z)\right)$ is the coefficient of $p^{m}$ in the expansion of $\eta(G(z, p))$ about $p$ as

$$
\eta\left(G\left(z, p, C_{k}\right)\right)=\eta_{0}\left(g_{0}(z)\right)+\sum_{m=1}^{\infty} \eta_{m}\left(g_{0}, g_{1}, g_{2}, \ldots, g_{m}\right) p^{m}
$$

The convergence of the series (36) depends upon $C_{k}$. For convergence at $p=1$, the $r$ th-order approximation $\widetilde{G}$ is

$$
\widetilde{G}\left(z, C_{1}, C_{2}, \ldots, C_{r}\right)=g_{0}(z)+\sum_{j=1}^{r} g_{j}\left(z, C_{1}, C_{2}, \ldots, C_{j}\right) .
$$

Substituting (43) into (31), the expression for the residual is

$$
R\left(z, C_{1}, C_{2}, \ldots, C_{r}\right)=\left\{\begin{array}{l}
\zeta\left(\widetilde{G}\left(z, C_{1}, C_{2}, \ldots, C_{r}\right)\right)+f(z) \\
+\eta\left(\widetilde{G}\left(z, C_{1}, C_{2}, \ldots, C_{r}\right)\right) .
\end{array}\right.
$$

$\widetilde{G}$ will be the exact solution if $R=0$, but in case of nonlinearity, it does not happen generally. To find the optimal values of the constants $C_{k}$, there are different methods to apply. We apply the method of least square as follows:

$$
J=\int_{a}^{b} R^{2}\left(z, C_{1}, C_{2}, \ldots, C_{r}\right) d z .
$$

Minimizing this function, we have

$$
\frac{\partial J}{\partial C_{i}}\left(z, C_{1}, C_{2}, \ldots, C_{r}\right)=0, \quad i=1,2,3, \ldots, r,
$$

where $a$ and $b$ are chosen in the domain of the problem for locating the suitable $C_{k}^{\prime} s(k=1,2, \ldots, r)$. The approximate solution is well determined for these values of $C_{k}$.

\section{Application of OHAM to Our Problem}

Using $m=1, R=1$, and $\gamma=1$ in (11), we have the following. Zeroth-order problem is

$$
\begin{gathered}
G_{0}^{i v}(x)=0, \\
G_{0}(0)=0, \quad G_{0}^{\prime \prime}(0)=0, \\
G_{0}(1)=1, \quad G_{0}^{\prime}(1)=G_{0}^{\prime \prime}(1) .
\end{gathered}
$$

First-order problem is

$$
\begin{gathered}
G_{1}^{(i v)}(z)=C_{1} G_{0}(z) G_{0}^{\prime \prime \prime}(z)+G_{0}^{(i v)}(z) \\
+C_{1} G_{0}^{(i v)}(z)-C_{1} G_{0}^{\prime \prime}(z), \\
G_{0}(0)=0, \quad G_{0}^{\prime \prime}(0)=0, \\
G_{0}(1)=0, \quad G_{0}^{\prime}(1)=G_{0}^{\prime \prime}(1) .
\end{gathered}
$$

Second-order problem is

$$
\begin{aligned}
& G_{2}^{(i v)}(z) \\
& =\left\{\begin{array}{c}
C_{2} G_{0}(z) G_{0}^{\prime \prime \prime}(z)+C_{1} G_{1}(z) G_{0}^{\prime \prime \prime}(z) \\
+C_{1} G_{0}(z) G_{1}^{\prime \prime \prime}(z)+C_{2} G_{0}^{(i v)}+G_{1}^{(i v)}(z) \\
+C_{1} G_{1}^{(i v)}(z)-C_{2} G_{0}^{\prime \prime}(z)-C_{1} G_{1}^{\prime \prime}(z), \\
G_{0}(0)=0, \quad G_{0}^{\prime \prime}(0)=0, \\
G_{0}(1)=0, \quad G_{0}^{\prime}(1)=G_{0}^{\prime \prime}(1) .
\end{array}\right.
\end{aligned}
$$


TABLE 1: DTM and OHAM solutions along with the residuals.

\begin{tabular}{lcccc}
\hline$z$ & DTM solution & DTM residual & OHAM solution & OHAM residual \\
\hline 0.0 & 0.0 & 0.0 & 0.0 & 0.0 \\
0.1 & 0.075739 & $-1.388 \times 10^{-17}$ & 0.075739 & $8.743 \times 10^{-10}$ \\
0.2 & 0.152935 & $-6.690 \times 10^{-14}$ & 0.152935 & $2.380 \times 10^{-8}$ \\
0.3 & 0.233046 & $-1.315 \times 10^{-11}$ & 0.233046 & $6.620 \times 10^{-8}$ \\
0.4 & 0.317540 & $-5.613 \times 10^{-10}$ & 0.317540 & $9.255 \times 10^{-8}$ \\
0.5 & 0.407893 & $-1.039 \times 10^{-8}$ & 0.407893 & $5.635 \times 10^{-8}$ \\
0.6 & 0.505591 & $-1.135 \times 10^{-7}$ & 0.505591 & $-4.382 \times 10^{-8}$ \\
0.7 & 0.612134 & $-8.630 \times 10^{-7}$ & 0.612134 & $-9.531 \times 10^{-8}$ \\
0.8 & 0.729034 & $-5.032 \times 10^{-6}$ & 0.729034 & $1.498 \times 10^{-7}$ \\
0.9 & 0.857813 & $-2.397 \times 10^{-5}$ & 0.857813 & $9.949 \times 10^{-7}$ \\
1.0 & 1.0 & $-9.743 \times 10^{-5}$ & 1.0 & $2.892 \times 10^{-6}$ \\
\hline
\end{tabular}

TABLE 2: Residuals for different values of $\gamma$ using DTM.

\begin{tabular}{lcccc}
\hline \multicolumn{1}{r}{$\gamma=0.5$} & $\gamma=0.6$ & $\gamma=0.7$ & $\gamma=0.8$ & 0.0 \\
\hline 0.0 & 0.0 & 0.0 & $-2.78 \times 10^{-17}$ & 0.0 \\
$3.75 \times 10^{-15}$ & $3.61 \times 10^{-16}$ & 0.0 & $-1.05 \times 10^{-13}$ & $-1.39 \times 10^{-17}$ \\
$3.10 \times 10^{-11}$ & $2.60 \times 10^{-12}$ & $1.42 \times 10^{-13}$ & $-2.12 \times 10^{-11}$ & $-6.69 \times 10^{-14}$ \\
$5.99 \times 10^{-9}$ & $4.97 \times 10^{-10}$ & $2.51 \times 10^{-11}$ & $-9.40 \times 10^{-10}$ & $-1.32 \times 10^{-11}$ \\
$2.50 \times 10^{-7}$ & $2.04 \times 10^{-8}$ & $9.09 \times 10^{-10}$ & $-1.82 \times 10^{-8}$ & $-5.61 \times 10^{-10}$ \\
$4.51 \times 10^{-6}$ & $3.59 \times 10^{-7}$ & $1.31 \times 10^{-8}$ & $-2.10 \times 10^{-7}$ & $-1.04 \times 10^{-8}$ \\
$4.76 \times 10^{-5}$ & $3.66 \times 10^{-6}$ & $9.43 \times 10^{-8}$ & $-1.69 \times 10^{-6}$ & $-1.14 \times 10^{-7}$ \\
$3.48 \times 10^{-4}$ & $2.57 \times 10^{-5}$ & $2.98 \times 10^{-7}$ & $-1.04 \times 10^{-5}$ & $-8.63 \times 10^{-7}$ \\
$1.94 \times 10^{-3}$ & $1.36 \times 10^{-4}$ & $-9.58 \times 10^{-7}$ & $-5.28 \times 10^{-5}$ & $-5.03 \times 10^{-6}$ \\
$8.76 \times 10^{-3}$ & $5.76 \times 10^{-4}$ & $1.84 \times 10^{-5}$ & $-2.28 \times 10^{-4}$ & $-2.40 \times 10^{-5}$ \\
$3.36 \times 10^{-2}$ & $2.04 \times 10^{-3}$ & $1.34 \times 10^{-4}$ & $-9.74 \times 10^{-5}$ \\
\hline
\end{tabular}

Third-order problem is

$$
\begin{aligned}
& G_{3}^{(i v)}(z) \\
& =\left\{\begin{array}{l}
C_{2} G_{1}(z) G_{0}^{\prime \prime \prime}(z)+C_{1} G_{2}(z) G_{0}^{\prime \prime \prime}(z) \\
+C_{2} G_{0}(z) G_{1}^{\prime \prime \prime}(z)+C_{1} G_{1}(z) G_{1}^{\prime \prime \prime}(z) \\
+C_{1} G_{0}(z) G_{2}^{\prime \prime \prime}(z)+C_{2} G_{1}^{(i v)}(z) \\
+G_{2}^{(i v)}(z)+C_{1} G_{2}^{(i v)}(z) \\
-C_{2} G_{1}^{\prime \prime}(z)+C_{1} G_{2}^{\prime \prime}(z), \\
G_{0}(0)=0, \quad G_{0}^{\prime \prime}(0)=0, \\
G_{0}(1)=0, \quad G_{0}^{\prime}(1)=G_{0}^{\prime \prime}(1) .
\end{array}\right.
\end{aligned}
$$

Fourth-order problem is

$$
\begin{aligned}
& G_{4}^{(i v)}(z) \\
& =\left\{\begin{array}{l}
C_{2} G_{2}(z) G_{0}^{\prime \prime \prime}(z)+C_{1} G_{3}(z) G_{0}^{\prime \prime \prime}(z) \\
+C_{2} G_{1}(z) G_{1}^{\prime \prime \prime}(z)+C_{1} G_{2}(z) G_{1}^{\prime \prime \prime}(z) \\
-C_{2} G_{0}(z) G_{2}^{\prime \prime \prime}(z)+C_{1} G_{1}(z) G_{2}^{\prime \prime \prime}(z) \\
+C_{1} G_{0}(z) G_{3}^{\prime \prime \prime}(z)+C_{2} G_{2}^{(i v)}(z)+G_{3}^{(i v)}(z) \\
+C_{1} G_{3}^{(i v)}(z)-C_{2} G_{2}^{\prime \prime}(z)-C_{1} G_{3}^{\prime \prime}(z), \\
G_{0}(0)=0, \quad G_{0}^{\prime \prime}(0)=0, \\
G_{0}(1)=0, \quad G_{0}^{\prime}(1)=G_{0}^{\prime \prime}(1) .
\end{array}\right.
\end{aligned}
$$

Fifth-order problem is

$$
\begin{aligned}
& G_{5}^{(i v)}(z) \\
& =\left\{\begin{array}{l}
C_{2} G_{3}(z) G_{0}^{\prime \prime \prime}(z)+C_{1} G_{4}(z) G_{0}^{\prime \prime \prime}(z) \\
+C_{2} G_{2}(z) G_{1}^{\prime \prime \prime}(z)+C_{1} G_{3}(z) G_{1}^{\prime \prime \prime}(z) \\
+C_{2} G_{1}(z) G_{2}^{\prime \prime \prime}(z)+C_{1} G_{2}(z) G_{2}^{\prime \prime \prime}(z) \\
+C_{2} G_{0}(z) G_{3}^{\prime \prime \prime}(z)+C_{1} G_{1}(z) G_{3}^{\prime \prime \prime}(z) \\
+C_{1} G_{0}(z) G_{4}^{\prime \prime \prime}(z)+C_{2} G_{3}^{(i v)}(z)+G_{4}^{(i v)}(z) \\
+C_{1} G_{4}^{(i v)}(z)-C_{2} G_{3}^{\prime \prime}(z)-C_{1} G_{4}^{\prime \prime}(z), \\
G_{0}(0)=0, \quad G_{0}^{\prime \prime}(0)=0, \\
G_{0}(1)=0, \quad G_{0}^{\prime}(1)=G_{0}^{\prime \prime}(1) .
\end{array}\right.
\end{aligned}
$$

By considering the fifth-order solution, we have

$$
\widetilde{G}(z)=\sum_{i=0}^{5} G_{i}\left(z, C_{1}, C_{2}\right) .
$$

The residual of the problem is

$$
\Re=\widetilde{G}^{(i v)}(z)-m^{2} \widetilde{G}^{\prime \prime}(z)+R \widetilde{G}(z) \widetilde{G}^{\prime \prime \prime}(z) .
$$




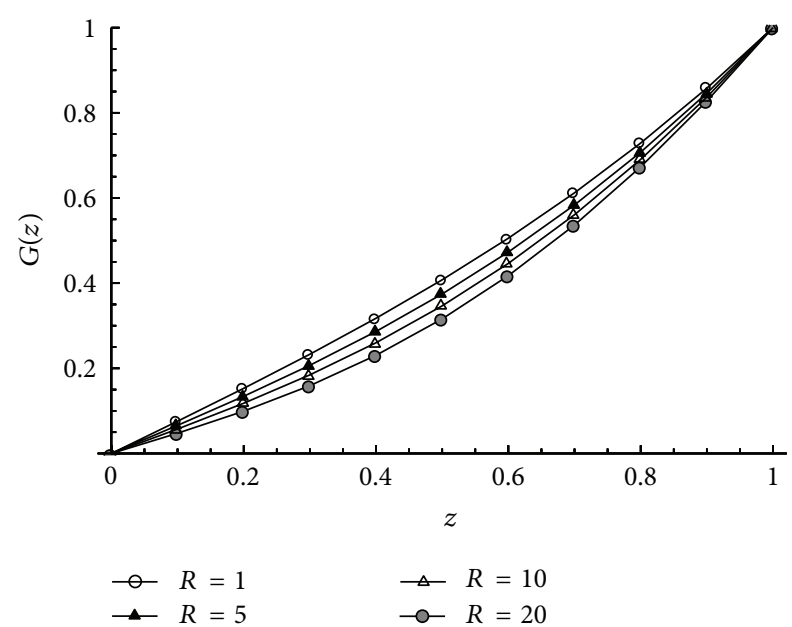

Figure 2: Plot shows the Graph of $G(z)$ for different values of $R$.

In order to find $C_{1}, C_{2}$, we apply the method of least square as follows:

$$
\begin{gathered}
J\left(C_{1}, C_{2}\right)=\int_{0}^{1} \mathfrak{R}^{2}\left(z, C_{1}, C_{2}\right) d z, \\
\frac{\partial J}{\partial C_{i}}\left(z, C_{1}, C_{2}\right)=0, \quad i=1,2 .
\end{gathered}
$$

Solving (56) for $C_{1}, C_{2}$, we get

$$
C_{1}=-0.925334, \quad C_{2}=-0.000937442 .
$$

Using these values of $C_{1}, C_{2}$, the approximate solution is given by:

$$
\begin{aligned}
& G(z)= \\
& \left\{\begin{array}{l}
0.754967 z+0.242564 z^{3}+0.00297182 z^{5} \\
-0.000509764 z^{7}+3.904619485 \times 10^{-6} z^{9} \\
+3.15747382 \times 10^{-6} z^{11}-1.21591 \times 10^{-7} z^{13} \\
-2.129279763 \times 10^{-8} z^{15}+1.398343 \times 10^{-9} z^{17} \\
+1.55939582 \times 10^{-10} z^{19}
\end{array}\right.
\end{aligned}
$$

As $p \in[0,1]$, the components of $G(z)$ vanish when going onward. In Table 1 , the solutions obtained by OHAM and DTM along with their residual are presented. Table 2 is constructed to discuss residuals for different values of $\gamma$.

\section{Numerical Results and Discussion}

Here we analyzed, under a transverse magnetic field, the squeezing fluid flow between two parallel infinite plates. DTM and OHAM are applied to obtain the solution of reduced nonlinear boundary value problem. The convergence region can easily be adjusted using OHAM as compared to DTM in this case. Differential transform method which is the straight forward application of Taylor's series suffers in general and particularly in initial value problems. Beside this fact, DTM leads easily to closed form solution. OHAM does

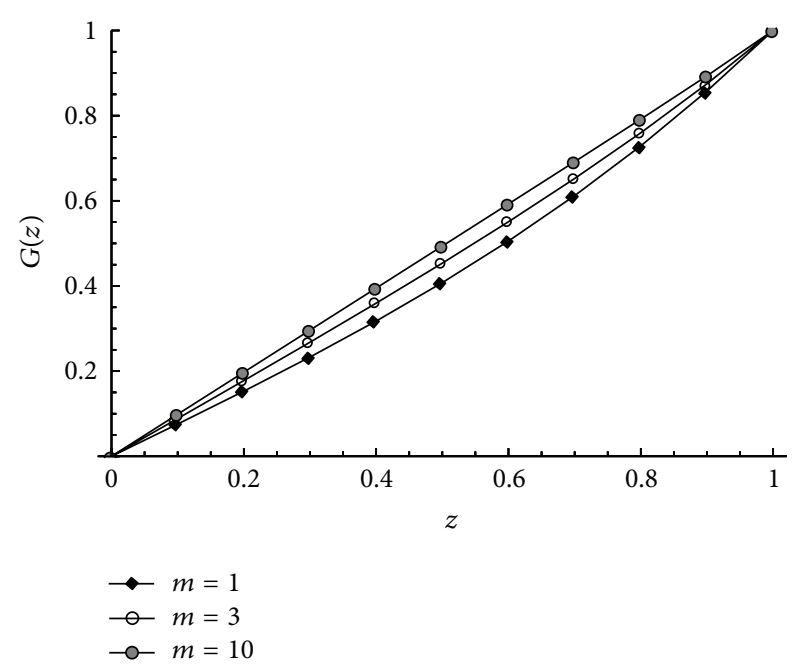

Figure 3: Analysis of $G(z)$ for different values of $m$ when $\gamma \neq 0$.

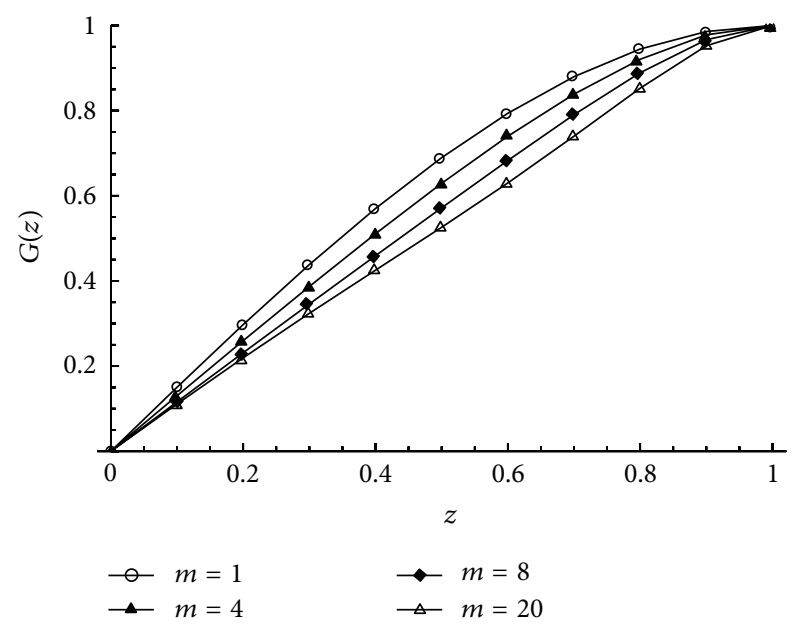

FIgURE 4: Analysis of $G(z)$ by varying $m$ when $\gamma=0$.

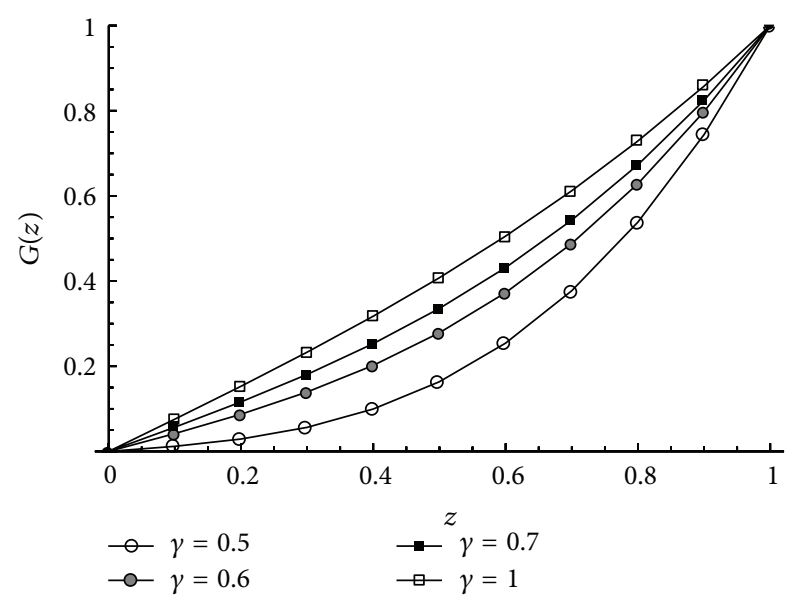

FIGURe 5: Analysis of $G(z)$ by Varying $\gamma$. 
not require discretization like other numerical and approximate methods. This technique requires less computational effort and the convergence of this method to the exact is fast enough. For computational purposes, we use Mathematica software.

In order to get an insight into the problem, it is important to discuss the influence of different flow parameters on the velocity profile $G(z)$ of the fluid.

(1) Figure 2 represents the influence of Reynold's number $R$ on the velocity of the fluid. It is observed from Figure 2 that by increasing $R$ the velocity of the fluid decreases.

(2) The influence of Hartmann number $m$ on the velocity of the fluid is shown in Figure 3. It is concluded that the velocity increases by increasing $m$ in case of $\gamma \neq 0$. However, from Figure 4, the velocity decreases due to an increase in $m$ for $\gamma=0$. This observation is in agreement with the literature (see [39, Figure 5]).

(3) Figure 5 shows the influence of the slip parameter $\gamma$ on the fluid velocity. By increasing $\gamma$, it is observed that the velocity of the fluid increases.

\section{References}

[1] T. C. Papanastasiou, G. C. Georgiou, and A. N. Alexandrou, Viscous Fluid Flow, CRC Press, 1994.

[2] M. J. Stefan, "Versuch Uber die scheinbare adhesion," Akademie Der Wissenschaften in Wien, vol. 69, article 713, 1874.

[3] Q. K. Ghori, M. Ahmed, and A. M. Siddiqui, "Application of homotopy perturbation method to squeezing flow of a newtonian fluid," International Journal of Nonlinear Sciences and Numerical Simulation, vol. 8, no. 2, pp. 179-184, 2007.

[4] X. J. Ran, Q. Y. Zhu, and Y. Li, "An explicit series solution of the squeezing flow between two infinite parallel plates," Communications in Nonlinear Science and Numerical Simulation, vol. 14, no. 1, pp. 119-132, 2007.

[5] R. J. Grimm, "Squeezing flows of Newtonian liquid films an analysis including fluid inertia," Applied Scientific Research, vol. 32, no. 2, pp. 149-166, 1976.

[6] W. F. Hughes and R. A. Elco, "Magnetohydrodynamic lubrication flow between parallel rotating disks," Journal of Fluid Mechanics, vol. 13, pp. 21-32, 1962.

[7] S. Kamiyama, "Inertia Effects in MHD hydrostatic thrust bearing," Transactions ASME, vol. 91, pp. 589-596, 1969.

[8] E. A. Hamza, "Magnetohydrodynamic squeeze film," Journal of Tribology, vol. 110, no. 2, pp. 375-377, 1988.

[9] S. Bhattacharyya and A. Pal, "Unsteady MHD squeezing flow between two parallel rotating discs," Mechanics Research Communications, vol. 24, no. 6, pp. 615-623, 1997.

[10] S. Islam, H. Khan, I. A. Shah, and G. Zaman, "An axisymmetric squeezing fluid flow between the two infinite parallel plates in a porous medium channel," Mathematical Problems in Engineering, vol. 2011, Article ID 349803, 10 pages, 2011.

[11] C.-L.-M.-H. Navier, "Sur les lois de l'équilibre et du mouvement des corps solides élastiques," Bulletin des Sciences par la Société Philomatique de Paris, pp. 177-181, 1823.
[12] C. le Roux, "Existence and uniqueness of the flow of secondgrade fluids with slip boundary conditions," Archive for Rational Mechanics and Analysis, vol. 148, no. 4, pp. 309-356, 1999.

[13] A. Ebaid, "Effects of magnetic field and wall slip conditions on the peristaltic transport of a Newtonian fluid in an asymmetric channel," Physics Letters A, vol. 372, no. 24, pp. 4493-4499, 2008.

[14] L. J. Rhooades, R. Resnic, T. O’ Bradovich, and S. Stegman, "Abrasive flow machining of cylinder heads and its positive effects on performance and cost characteristics," Tech. Rep., Dearborn, Mich, USA, 1996.

[15] T. Hayat, M. U. Qureshi, and N. Ali, “The inuence of slip on the peristaltic motion of third order fluid in an asymmetric channel," Physics Letters A, vol. 372, pp. 2653-2664, 2008.

[16] T. Hayat and S. Abelman, "A numerical study of the influence of slip boundary condition on rotating flow," International Journal of Computational Fluid Dynamics, vol. 21, no. 1, pp. 21-27, 2007.

[17] S. Abelman, E. Momoniat, and T. Hayat, "Steady MHD flow of a third grade fluid in a rotating frame and porous space," Nonlinear Analysis: Real World Applications, vol. 10, no. 6, pp. 3322-3328, 2009.

[18] A. H. Nayfeh, Problems in Perturbation, A Wiley-Interscience Publication, John Wiley \& Sons, New York, NY, USA, 1985.

[19] S. J. Liao, The proposed homotopy analysis technique for the solution of nonlinear problems [Ph.D. thesis], Shanghai Jiao Tong University, 1992.

[20] S. Liao, Beyond Perturbation. Introduction to the Homotopy Analysis Method, vol. 2 of CRC Series: Modern Mechanics and Mathematics, Chapman \& Hall/CRC, Boca Raton, Fla, USA, 2004.

[21] S. Liao, "On the homotopy analysis method for nonlinear problems," Applied Mathematics and Computation, vol. 147, no. 2, pp. 499-513, 2004.

[22] J.-H. He, "Homotopy perturbation method for solving boundary value problems," Physics Letters A, vol. 350, no. 1-2, pp. 87$88,2006$.

[23] J. H. He, "Application of homotopy perturbation method to nonlinear wave equations," Chaos, Solitons and Fractals, vol. 26, no. 3, pp. 695-700, 2005.

[24] J.-H. He, "Comparison of homotopy perturbation method and homotopy analysis method," Applied Mathematics and Computation, vol. 156, no. 2, pp. 527-539, 2004.

[25] V. Marinca, N. Herişanu, and I. Nemeş, "Optimal homotopy asymptotic method with application to thin film flow," Central European Journal of Physics, vol. 6, no. 3, pp. 648-653, 2008.

[26] V. Marinca and N. Herişanu, "Application of optimal homotopy asymptotic Method for solving nonlinear equations arising in heat transfer," International Communications in Heat and Mass Transfer, vol. 35, no. 6, pp. 710-715, 2008.

[27] J. Ali, S. Islam, S. Islam, and G. Zaman, "The solution of multipoint boundary value problems by the optimal homotopy asymptotic method," Computers \& Mathematics with Applications, vol. 59, no. 6, pp. 2000-2006, 2010.

[28] V. Marinca and N. Herisanu, "Optimal homotopy perturbation method for strongly nonlinear differential equations," Nonlinear Science Letters A, vol. 1, no. 3, pp. 273-280, 2010.

[29] J. K. Zhou, Deferential Transformation and Its Application for Electrical Circuits, Huazhong University Press, Wuhan, China, 1986.

[30] S. Çatal, "Solution of free vibration equations of beam on elastic soil by using differential transform method," Applied Mathematical Modelling, vol. 32, no. 9, pp. 1744-1757, 2008. 
[31] S. Çatal and H. H. Çatal, "Buckling analysis of partially embedded pile in elastic soil using differential transform method," Structural Engineering and Mechanics, vol. 24, no. 2, pp. 246269, 2006.

[32] S. Çatal, "Analysis of free vibration of beam on elastic soil using differential transform method," Structural Engineering and Mechanics, vol. 24, no. 1, pp. 51-63, 2006.

[33] S. Catal, "Response of forced Euler-Bernoulli beams using differential transform method," Structural Engineering and Mechanics, vol. 42, no. 1, pp. 95-119, 2012.

[34] F. Ayaz, "Applications of differential transform method to differential-algebraic equations," Applied Mathematics and Computation, vol. 152, no. 3, pp. 649-657, 2004.

[35] H. Liu and Y. Song, "Differential transform method applied to high index differential-algebraic equations," Applied Mathematics and Computation, vol. 184, no. 2, pp. 748-753, 2007.

[36] F. Ayaz, "On the two-dimensional differential transform method," Applied Mathematics and Computation, vol. 143, no. 2-3, pp. 361-374, 2003.

[37] M. R. Mohyuddin and T. Götz, "Resonance behaviour of viscoelastic fluid in Poiseuille flow in the presence of a transversal magnetic field," International Journal for Numerical Methods in Fluids, vol. 49, no. 8, pp. 837-847, 2005.

[38] S. H. Chang and I. L. Chang, "A new algorithm for calculating one-dimensional differ- ential transform of nonlinear functions," Applied Mathematics and Computation, vol. 195, no. 2, pp. 799-808, 2008.

[39] S. Islam, M. Ullah, G. Zaman, and M. Idrees, "Approximate solutions to MHD squeezing fluid flow," Journal of Applied Mathematics \& Informatics, vol. 29, no. 5-6, pp. 1081-1096, 2011. 


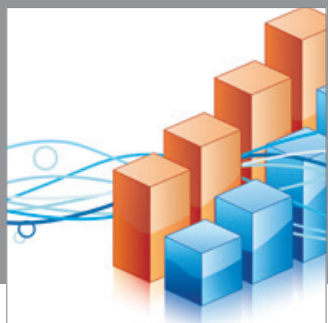

Advances in

Operations Research

mansans

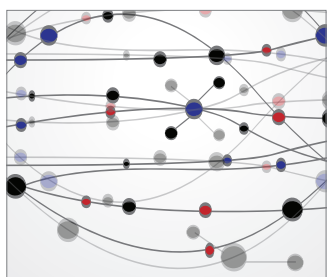

The Scientific World Journal
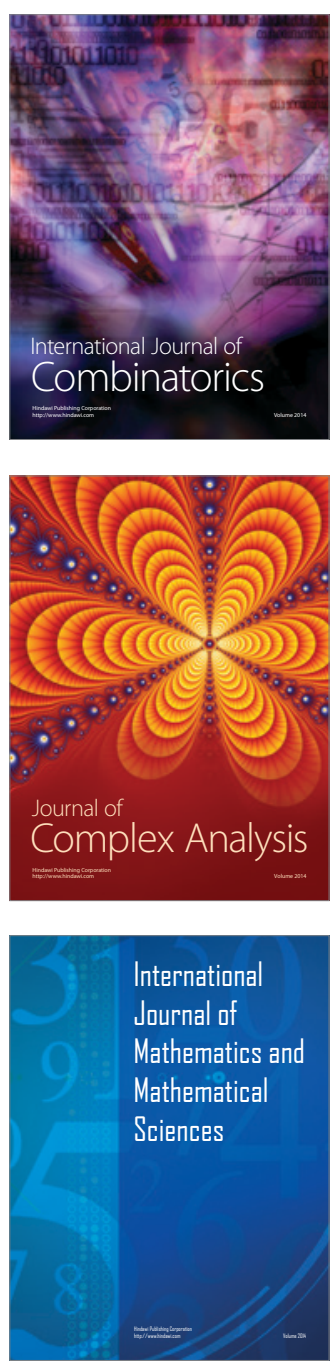
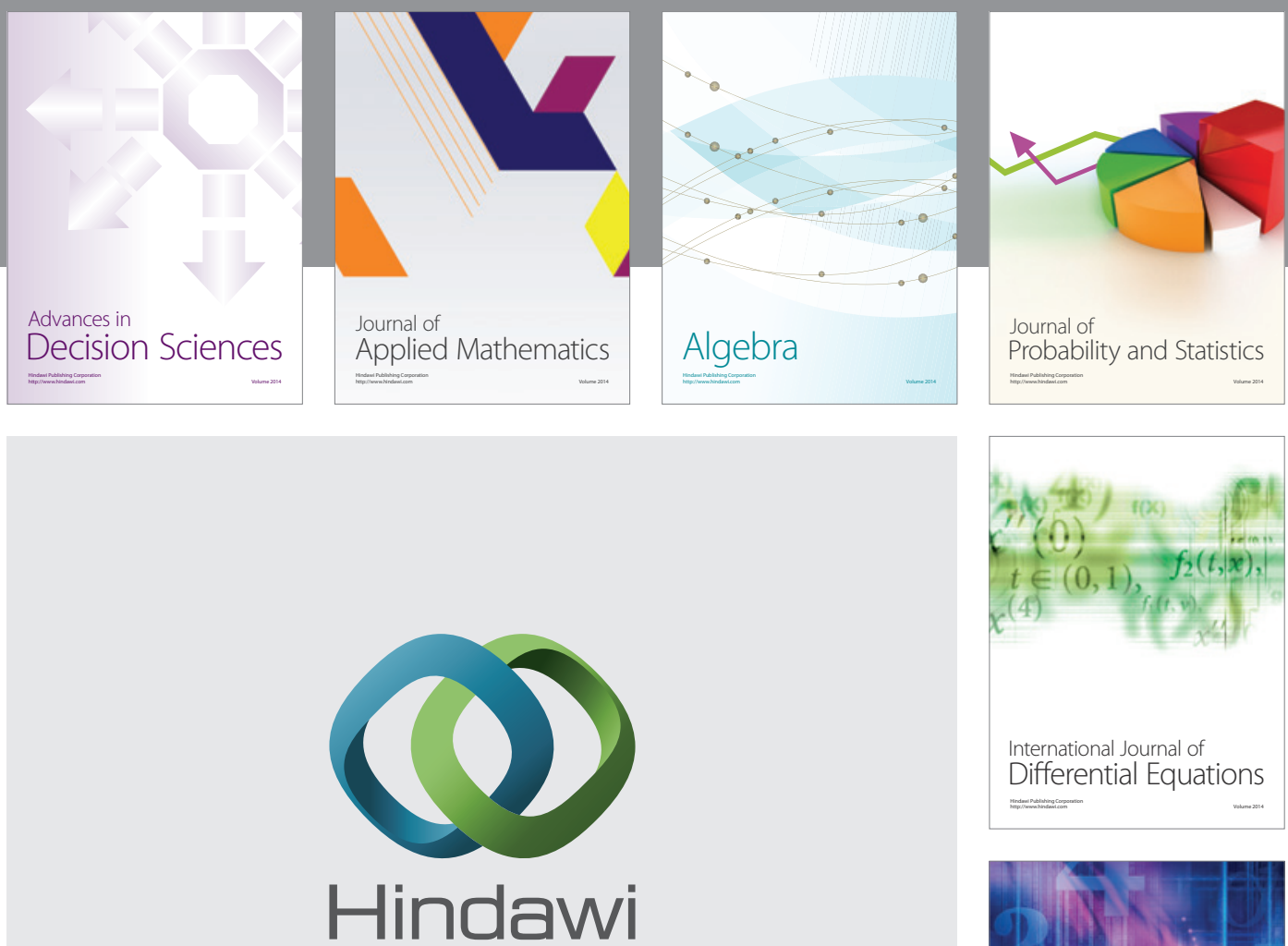

Submit your manuscripts at http://www.hindawi.com
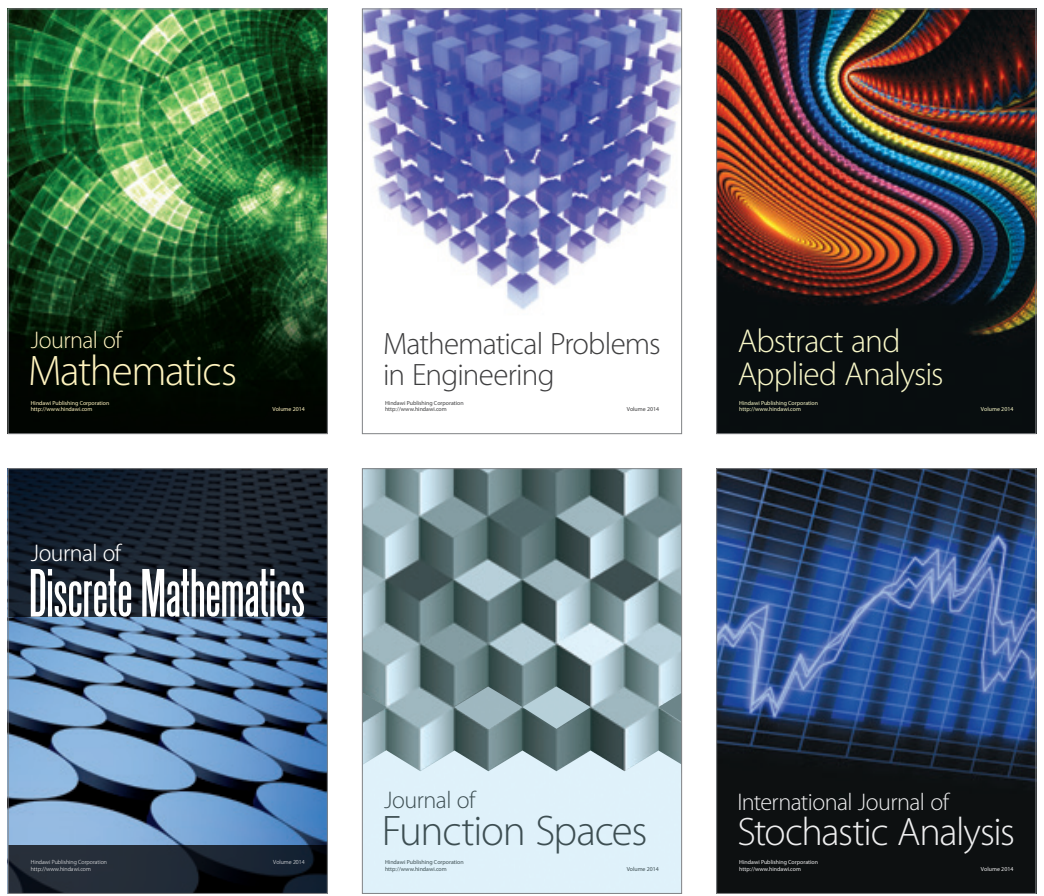

Journal of

Function Spaces

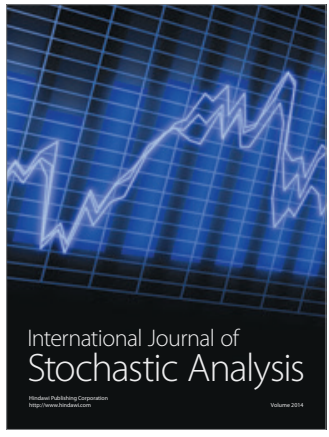

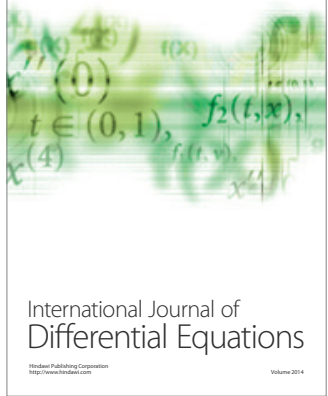
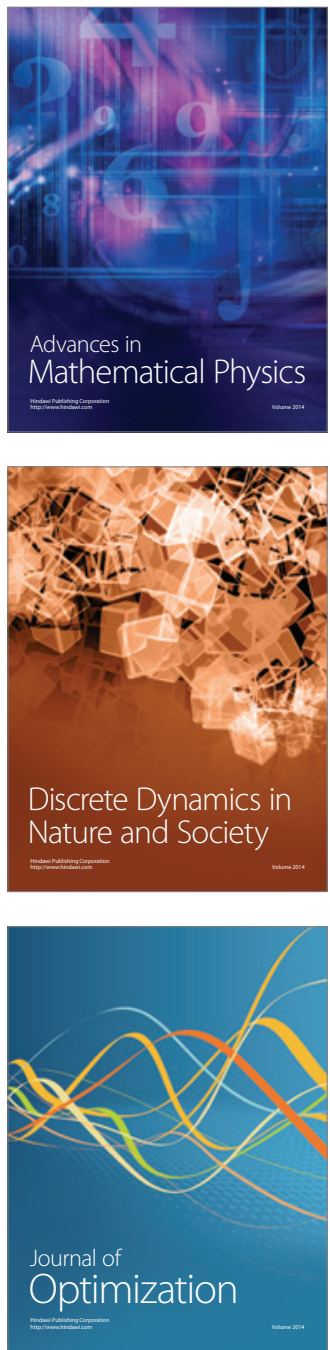